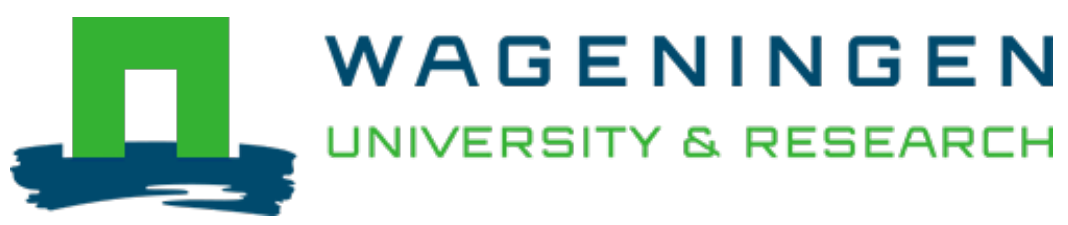

\title{
Levels of inflammation markers are associated with the risk of recurrence and all- cause mortality in patients with colorectal cancer
}

\author{
Cancer Epidemiology Biomarkers \& Prevention
}

Wesselink, Evertine; Balvers, Michiel G.J.; Kok, Dieuwertje E.; Winkels, Renate M.; Zutphen, Moniek et al https://doi.org/10.1158/1055-9965.EPI-20-1752

This article is made publicly available in the institutional repository of Wageningen University and Research, under the terms of article $25 \mathrm{fa}$ of the Dutch Copyright Act, also known as the Amendment Taverne. This has been done with explicit consent by the author.

Article 25 fa states that the author of a short scientific work funded either wholly or partially by Dutch public funds is entitled to make that work publicly available for no consideration following a reasonable period of time after the work was first published, provided that clear reference is made to the source of the first publication of the work.

This publication is distributed under The Association of Universities in the Netherlands (VSNU) 'Article $25 \mathrm{fa}$ implementation' project. In this project research outputs of researchers employed by Dutch Universities that comply with the legal requirements of Article $25 \mathrm{fa}$ of the Dutch Copyright Act are distributed online and free of cost or other barriers in institutional repositories. Research outputs are distributed six months after their first online publication in the original published version and with proper attribution to the source of the original publication.

You are permitted to download and use the publication for personal purposes. All rights remain with the author(s) and / or copyright owner(s) of this work. Any use of the publication or parts of it other than authorised under article $25 \mathrm{fa}$ of the Dutch Copyright act is prohibited. Wageningen University \& Research and the author(s) of this publication shall not be held responsible or liable for any damages resulting from your (re)use of this publication.

For questions regarding the public availability of this article please contact openscience.library@,wur.nl 


\section{Levels of Inflammation Markers Are Associated with the Risk of Recurrence and All-Cause Mortality in Patients with Colorectal Cancer}

Evertine Wesselink', Michiel G.J. Balvers', Dieuwertje E. Kok', Renate M. Winkels', Moniek van Zutphen', Ruud W.M. Schrauwen ${ }^{2}$, Eric T.P. Keulen ${ }^{3}$, Ewout A. Kouwenhoven ${ }^{4}$, Stephanie O. Breukink ${ }^{5,6}$, Renger F. Witkamp ${ }^{1}$, Johannes H.W. de Wilt ${ }^{7}$, Martijn J.L. Bours ${ }^{5}$, Matty P. Weijenberg ${ }^{5}$, Ellen Kampman', and Fränzel J.B. van Duijnhoven'

\section{ABSTRACT}

Background: We investigated whether preoperative and postoperative levels of inflammation markers, which have mechanistically been linked to colorectal cancer progression, were associated with recurrence and all-cause mortality in patients with colorectal cancer.

Methods: Data of two prospective cohort studies were used. For the current analysis, patients with stage I to III colorectal cancer were considered. Data on inflammation [IL6, IL8, IL10, TNFo, high-sensitivity C-reactive protein (hsCRP), and a combined inflammatory $z$-score] were available for 747 patients before surgery and for 614 patients after surgery. The associations between inflammation marker levels and colorectal cancer recurrence and all-cause mortality were examined using multivariable Cox proportional hazard regression models, considering patient characteristics and clinical and lifestyle factors.

Results: Higher preoperative and postoperative hsCRP levels were associated with a higher risk of recurrence $\left[\mathrm{HR}_{\text {per doubling }}\right.$

\section{Introduction}

Chronic low-grade inflammation is an important driver of cancer development and progression (1-4). This includes colorectal cancer $(2,5)$. Vice versa, NSAIDs and cytokine antagonist or antibodies may play a role in the prevention and treatment of colorectal cancer $(2,5,6)$. Together, this underlines the potential relevance of inflammation during tumor onset and progression.

The association between C-reactive protein (CRP), a nonspecific marker of inflammation, and colorectal cancer prognosis has been extensively studied (7-9). Higher CRP levels were associated with a

'Division of Human Nutrition and Health, Wageningen University \& Research, Wageningen, the Netherlands. ${ }^{2}$ Department of Gastroenterology, Bernhoven, Uden, the Netherlands. ${ }^{3}$ Department of Gastroenterology, Zuyderland Medical Centre, Sittard-Geleen, the Netherlands. ${ }^{4}$ Department of Surgery, Ziekenhuis Groep Twente, Almelo, the Netherlands. ${ }^{5}$ Department of Epidemiology, GROW-School for Oncology and Developmental Biology, Maastricht University, Maastricht, the Netherlands. ${ }^{6}$ Department of Surgery, Maastricht University Medical Center, Maastricht, the Netherlands. ${ }^{7}$ Department of Surgery, Radboud University Medical Centre, Nijmegen, the Netherlands.

Note: Supplementary data for this article are available at Cancer Epidemiology, Biomarkers \& Prevention Online (http://cebp.aacrjournals.org/).

Corresponding Author: Evertine Wesselink, Human Nutrition and Health, Wageningen University \& Research, Wageningen, 6709 WE, the Netherlands. E-mail: vera.wesselink@wur.nl

Cancer Epidemiol Biomarkers Prev 2021;30:1089-99

doi: 10.1158/1055-9965.EPI-20-1752

(C2021 American Association for Cancer Research.
(95\% CI), $1.15(1.02-1.30)$ and $1.34(1.16-1.55)]$ and all-cause mortality $\left[\mathrm{HR}_{\text {per doubling }}(95 \% \mathrm{CI}) 1.13\right.$ (1.01-1.28) and 1.15 (0.98-1.35)]. A doubling in IL8 levels (preoperative levels $\mathrm{HR}=1.23$; 95\% CI, 1.00-1.53 and postoperative levels $\mathrm{HR}=$ $1.61 ; 95 \% \mathrm{CI}, 1.23-2.12$ ) and a higher combined inflammatory $z$-score (preoperative $\mathrm{HR}_{\text {per doubling }}=1.39 ; 95 \% \mathrm{CI}, 1.03-1.89$ and postoperative $\mathrm{HR}_{\text {per doubling }}=1.56$; $95 \% \mathrm{CI}, 1.06-2.28$ ) were associated with a higher risk of all-cause mortality, but not recurrence. No associations between IL6, IL10, and TNF $\alpha$ and recurrence or all-cause mortality were observed.

Conclusions: Preoperative and postoperative levels of specific inflammation markers were associated with recurrence and/or allcause mortality.

Impact: The complex role of inflammation in cancer recurrence merits further elucidation by investigating local inflammation at the tumor site.

higher risk of recurrences (9) and a worse disease-free, cancer-specific, and overall survival $(7,8)$. Next to CRP, more specific inflammation markers have also been studied, for example, cytokines including several ILs and TNF $\alpha$, suggested crucial players in colorectal cancer tumor immunology $(2,5,10)$. Higher levels of IL6, TNF $\alpha$, CRP, and IL8 have been associated with more advanced colorectal cancer (11-15). Likewise, higher levels of IL6, IL8, IL10, and TNF $\alpha$ have also been associated with a worse disease-free, cancer-specific, and overall survival $(11,14,16-20)$.

So far, studies investigating potential associations between cytokines and colorectal cancer involved relatively small numbers of subjects, usually between 50 and 250. Importantly, those studies only measured cytokine levels at diagnosis and thus before surgery. The timing of measurements could be important, because cytokine levels might be different in the preoperative phase when the tumor is still present and there is active interaction with the tumor microenvironment and host immunity, this is not the case when the tumor is removed (5). In addition, previous studies did not adjust for lifestyle and other clinical factors not directly linked to the tumor itself. Several lifestyle factors or other patient characteristics, such as physical activity, BMI, smoking and dietary habits, comorbidities, NSAID use, and vitamin D status are of potential interest as they are associated with both inflammation as well as mortality (21-30).

In view of these knowledge gaps and considerations, we investigated preoperative as well as postoperative levels of IL6, IL8, IL10, TNF $\alpha$, and CRP levels in association with cancer recurrence and all-cause mortality, while taking lifestyle and clinical factors into account, in a large study of 747 patients with colorectal cancer. 


\section{Materials and Methods}

\section{Study design and population}

Data from two prospective cohort studies were used, the COLON study and the EnCoRe study. The design of the COLON study (ClinicalTrials.gov identifier:NCT03191110; ref. 31) and the EnCoRe study (trialregister.nl identifier:NL6904; ref. 32) have been described elsewhere. Briefly, in both studies, newly diagnosed patients with colorectal cancer were recruited directly after diagnosis and were followed during and after treatment. Men and women above the age of 18 were eligible. In the COLON study, patients with a first primary stage I to IV colorectal cancer were eligible, whereas patients with primary stage I to III colorectal cancer were eligible in the EnCoRe study. The COLON and EnCoRe studies were approved by regional Medical Ethics Committees (NL30446.091.09 and NL38786.068.11, respectively). All patients signed written informed consent.

In total 1,089 patients were recruited of which 791 in the COLON study and 298 in the EnCoRe study (Fig. 1). For 232 patients, no preoperative blood samples were available, mainly because of the small time-window between diagnosis, study inclusion, and surgery. Patients with missing data about stage of disease $(n=57)$ or who had stage IV disease $(n=52)$ were also excluded. In total, 747 patients were included in the analyses of the association between preoperative levels and all-cause mortality. For 133 patients, data on inflammation markers was missing 6 months after diagnosis (post-). For the asso- ciation between postoperative levels and all-cause mortality, 614 patients were included. Data about recurrence were missing for 7 patients for the preoperative analyses and for 5 patients for the postoperative analyses.

\section{Blood collection}

In principle, blood samples were obtained during a regular clinical visit to the hospital at diagnosis and 6 months after diagnosis (median $=5.8 ;$ IQR $=5.4-6.4$ months) in the COLON study and during a regular clinical visit to the hospital or by a research assistant during a home visit before start of cancer treatment and 6 weeks and 6 months after cancer treatment in the EnCoRe study. To be able to harmonize the data of both cohorts for analyses after colorectal cancer diagnosis, we selected the time point in the EnCoRe cohort closest to 6 months after diagnosis (median $=6.9$; IQR $=6.4-8.5$ months).

All blood samples were centrifuged and aliquoted and plasma was stored at $-80^{\circ} \mathrm{C}$ until further analysis.

\section{Inflammation markers}

The Pro-inflammatory Panel 1 (Human) Kit from MSD cytokine multiplex assays (Meso Scale Diagnostics) was used to measure IL6, IL8, IL10, and TNF $\alpha$ in plasma in our lab at Wageningen University \& Research. These inflammatory markers has been selected on the basis of their suggested role in colorectal cancer progression (29).

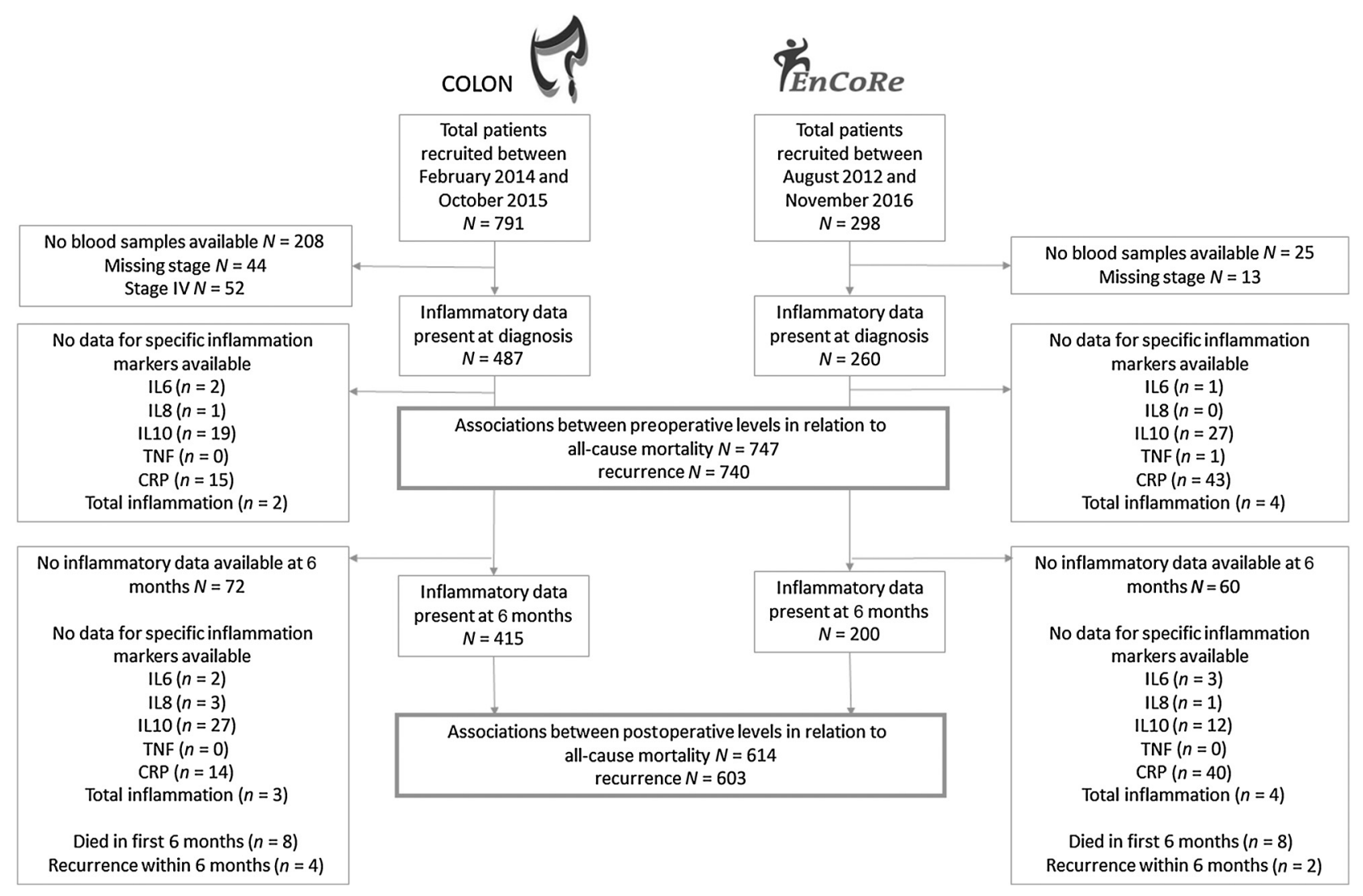

Figure 1.

Flowchart of the participants included in this study. Note that the number of the participants included in the analyses for the association between a specific inflammation marker and recurrence or all-cause mortality could be slightly different per inflammation marker, due to missing values in specific inflammation markers. 
Measurements of cytokines were done in duplicate according to the manufacturer's protocol. Highest inter and intra-batch coefficients of variation for the manufacturer's quality controls for all cytokines were $<8 \%$, and reported values deviated no more than $15 \%$ from the assigned target values. Detailed information about the analytical methods has been published previously (30). High-sensitive C-reactive protein (hsCRP) was measured using an immuno-MALDI mass spectrometry method (BEVITAL; ref. 33). The interassay coefficient ranged between $3 \%$ and $6 \%$.

A combined inflammatory $z$-score (including hsCRP, TNF, IL6, IL8, and IL10) was calculated (34) to cluster conceptually related markers of low-grade inflammation and improve statistical efficiency. First, a normalized $z$-score for each individual biomarker was calculated. The combined score was calculated by summing the $z$-scores of each individual marker and dividing the total sum by the number of inflammation markers included. If data of two or more inflammatory markers were missing for a patient $(n=6$ preoperative and $n=7$ postoperative), no combined inflammatory $z$-score was calculated.

\section{Colorectal cancer outcomes}

Information on recurrence was collected from medical records by the Dutch Cancer Registration. Recurrence was defined as a local regional recurrence or distant metastasis. Follow-up time for recurrence was calculated starting from the date of blood collection, either preoperative or postoperative, until the date of recurrence or until the date recurrence status was updated (COLON: February 2018; EnCoRe: March 2018), or the date of end of follow-up, whichever came first.

Information on all-cause mortality was gathered from linkage with the Municipal Personal Record Database. Follow-up time was defined starting from the date of blood collection, either preoperative or postoperative, until the date of death, or until the last date survival status was updated (COLON: December 2019; EnCoRe: May 2019), whichever came first.

\section{Covariate assessment}

Information on demographics, smoking, physical activity, diet, use of dietary supplements, and NSAIDs was obtained using selfadministered questionnaires. Physical activity was assessed using the Short Questionnaire to Assess Health-enhancing physical activity (SQUASH; ref. 35). Information on height, weight, and waist and hip circumference was collected using self-administered questionnaires in the COLON study. In the EnCoRe study, these measurements were performed by trained research dieticians during home visits. Habitual dietary intake in the month (COLON) or year (EnCoRe) preceding diagnosis was assessed using a semiquantitative food frequency questionnaire (FFQ). The FFQ used in the COLON study consists of 204 items. The FFQ used in the EnCoRe study consists of 253 items. Six months after diagnosis, habitual dietary intake was assessed with a FFQ in the COLON study and a 7-day food diary in the EnCoRe study.

Clinical factors, such as stage of disease, tumor location, tumor differentiation, date of start treatment, type of treatment, and presence of comorbidities were derived from the Dutch ColoRectal Audit (DCRA; ref. 36).

For both cohorts, serum 25 -hydroxy vitamin $\mathrm{D}_{3}\left[25(\mathrm{OH}) \mathrm{D}_{3}\right]$ levels were measured by LC/MS-MS in the Canisius Wilhelmina Hospital, Nijmegen, the Netherlands (13).

\section{Data analyses}

This analysis included 747 stage I to III patients with colorectal cancer of whom data of levels of inflammation markers was available at diagnosis. For 614 patients, data about levels of inflammation 6 months after diagnosis was available.

Population characteristics are described as numbers with percentages and medians with interquartile range (IQR).

IL6, IL8, IL10, TNFo, hsCRP, and the combined inflammatory $z$-score were the exposure variables in this study. Inflammation markers were $\log 2$ transformed to obtain normally distributed data. HRs from continuous analyses should therefore be interpreted as the increased risk with a doubling in the levels of an inflammation marker.

To visualize the relationship between inflammation and colorectal cancer outcomes, restricted cublic splines (RCS) was used (SAS Macro \%RCS_Reg vs1.44). Three knots were placed on the 10th, 50th, and 90th percentile, where the 50th percentile was used as the reference. The associations between the majority of inflammation markers and outcomes were linear (Supplementary Fig. S1). Therefore, the association between continuous inflammation markers and colorectal cancer outcomes was investigated using Cox proportional hazard models.

First, a crude Cox proportional hazard model, including only the main exposure of interest (i.e., IL6, IL8, IL10, TNFo, hsCRP, or the combined inflammatory $z$-score) and the outcome (i.e., recurrence or all-cause mortality) was run (model 1). Second, based on literature, analyses were adjusted for age, sex, BMI, stage of disease (I/II/III), season of blood collection, and cohort (model 2; refs. 7, 9, 37). In addition, other clinical and lifestyle factors were investigated for confounding; tumor location (colon/rectal), differentiation grade (good/moderate, bad), daily use of NSAIDs (yes/no), daily use of statins (yes/no), having comorbidities (yes/no), received treatment (only surgery/surgery and chemotherapy/surgery and radiotherapy/ surgery and chemoradiation), neo-adjuvant treatment (yes/no), adjuvant treatment (yes/no), number of days between surgery and postoperative blood sampling, hours of moderate to vigorous physical activity per week (continuous), smoking habits (current/former/never), education level (low/medium/high), intake of red and processed meat $(\mathrm{g} /$ day), intake of vegetables and fruits ( $\mathrm{g} /$ day), fiber intake (g/day), and alcohol intake (g/day). Only smoking habits changed the association of model 2 with more than $10 \%$ and this was added to the fully adjusted model (model $2+$ smoking).

Above-mentioned associations were examined with data from two time points: preoperative (at diagnosis, in $92 \%$ of the participants before the start of treatment) and postoperative ( 6 months after diagnosis, $83 \%$ after the end of treatment). Confounding by modifiable lifestyle factors such as dietary intake, physical activity levels, and BMI was evaluated using data assessed at the same time-point as blood measurements were performed. Correlations between preoperative and postoperative levels of inflammatory markers were examined using linear regression analyses.

Sensitivity analyses were done: (i) for NSAID nonusers only; (ii) for nonsmokers only; (iii) excluding patients who received (neo)adjuvant treatment (for preoperative levels patients who received neoadjuvant treatment were excluded; for postoperative levels patients who received adjuvant treatment were excluded; (iv) excluding patients still undergoing treatment (for preoperative levels patients who already started treatment were excluded; for postoperative levels patients who still underwent adjuvant treatment were excluded; (v) excluding outliers of inflammatory markers ( $>3 \mathrm{SD}$ above the mean); (vi) excluding older blood samples (levels measured after 2 years of storage, since cytokines were previously shown to remain stable in plasma for a period up to 2 years of storage at $-80^{\circ} \mathrm{C}$; ref. 38); (vii) correction for preoperative or postoperative levels; and (viii) excluding patients with hsCRP levels $>10 \mathrm{mg} / \mathrm{mL}$. 
To further investigate the interference of conditions influencing inflammation, such as other comorbidities $(27,28)$ and vitamin D status $(29,30)$, we tested for multiplicative interaction between inflammation and having any comorbidities, specifically cardiometabolic comorbidities and vitamin D status in relation to colorectal cancer outcomes. Multiplicative interaction was assessed using the interaction term approach. Thus, the exposure (inflammation marker levels) and the above-mentioned potential effect modifiers were entered as a product term in the model, for example, IL6*vitamin status. We did not stratify our analyses on vitamin D status and comorbidities, since all $P$ for interaction values were above 0.05 . The only exception was the interaction between preoperative TNF $\alpha$ levels, vitamin D status, and colorectal cancer recurrence $P=0.005$. However, although statistically significant, this finding might be a chance finding, therefore we did not stratify analyses for vitamin D status.

All statistical analyses were performed in SAS, version 9.4. $P$ values $<0.05$ were considered statistically significant.

\section{Results}

\section{Population characteristics}

Mean age of the total population was $67(\mathrm{IQR}=62-73)$ years, $33 \%$ was female and $44 \%$ of the patients were diagnosed with stage III of disease (Table 1). Compared with patients with a low combined inflammatory $z$-score (tertile 1), patients with a high combined inflammatory z-score (tertile 3 ) were older, had a higher BMI, more often had a low education level, were less physically active, more often had colon cancer, were more often in stage II of disease, more often had comorbidities and had lower levels of $25(\mathrm{OH}) \mathrm{D}_{3}$ (Table $\mathbf{1}$ ).

Postoperative samples were obtained 5 to 6 months after surgery in the majority of patients (median $=174$ days; IQR $=151-194$ ). A moderately strong correlation between pre-operative and postoperative inflammatory marker levels was observed (IL6, $r=0.4$; IL8, $r=0.6$; IL10, $r=0.4$; TNF $\alpha, r=0.6$; hSCRP, $r=0.4$, combined inflammatory $z$-score, $r=0.4)$. Levels of inflammation markers did not change substantially ( $>1 \mathrm{SD}$ ) between diagnosis and 6 months after diagnosis in $77 \%$ of our study population. In $13 \%$ of the study population levels increased with more than $1 \mathrm{SD}$, whereas in $10 \%$ levels decreased with more than 1SD. Characteristics of patients with increased, stable, and decreased levels are depicted in Supplementary Table S1.

During a median follow-up of 3.0 years $(\mathrm{IQR}=2.4-3.5)$ for recurrence and 4.7 years $(\mathrm{IQR}=4.2-5.3)$ for all-cause mortality, 88 recurrences and 98 deaths occurred. For the postoperative measurements, the median follow-up was 2.5 years $(\mathrm{IQR}=2.0-3.0)$ for recurrence and 4.3 years $(\mathrm{IQR}=3.8-4.90)$ for all-cause mortality, in which 64 recurrences and 61 deaths occurred.

Inflammation markers, recurrence, and all-cause mortality

A doubling in preoperative hsCRP levels was associated with a $15 \%$ higher risk of colorectal cancer recurrence ( $\mathrm{HR}=1.15$; 95\% CI, 1.021.30 ) and a doubling in postoperative hsCRP levels was associated with a $34 \%$ higher risk of colorectal cancer recurrence $(\mathrm{HR}=1.34 ; 95 \% \mathrm{CI}$, 1.16-1.55). Doubling of preoperative as well as postoperative cytokine levels (IL6, IL8, IL10, and TNF $\alpha$ ) and the combined inflammatory z-score were not associated with cancer recurrence (Fig. 2).

For all-cause mortality, preoperative as well as postoperative levels of proinflammatory markers, that is, IL6, IL8, TNFo, and hsCRP, tended to be associated with a higher risk (Fig. 2). These associations were statistically significant for both preoperative and postoperative levels of IL8 ( $\mathrm{HR}=1.23$; 95\% CI, $1.00-1.53$ and $\mathrm{HR}=1.61 ; 95 \% \mathrm{CI}$, $1.23-2.12$, respectively) and the combined inflammatory $z$-score
$(\mathrm{HR}=1.39 ; 95 \% \mathrm{CI}, 1.03-1.89$ and $\mathrm{HR}=1.56 ; 95 \% \mathrm{CI}, 1.06-2.28$, respectively) and for preoperative levels of hsCRP $(\mathrm{HR}=1.13 ; 95 \% \mathrm{CI}$, 1.01-1.28). Levels of IL10 were not associated with all-cause mortality.

\section{Results of sensitivity analyses}

No differences in associations with recurrence and all-cause mortality were observed when non-NSAID users, nonsmokers, patients receiving no treatment at the moment of blood collection, and patients not receiving (neo)adjuvant treatment were compared with the total population (Figs. 3 and 4). Also, no differences were observed when excluding old blood samples or outliers. Associations between postoperative levels of inflammation markers and recurrence and all-cause mortality did not markedly change after adjustment for preoperative levels. The association between preoperative levels and colorectal cancer outcomes diminished after correction for postoperative levels. No differences were observed for the associations between cytokines and colorectal cancer outcomes and hsCRP and recurrence when excluding patients with acute infections (hsCRP $>10 \mu \mathrm{g} / \mathrm{mL}$ ). However, the association between postoperative hsCRP levels and all-cause mortality disappeared.

\section{Discussion}

Associations between preoperatively and postoperatively analyzed inflammation marker levels and colorectal cancer outcomes were comparable. A doubling in hsCRP levels was associated with a higher risk of recurrence. No associations between IL6, IL8, IL10, $\mathrm{TNF} \alpha$, and the combined inflammatory $z$-score and recurrence were observed. Higher levels of IL8, hsCRP, and the combined inflammatory $z$-score were associated with a higher risk of all-cause mortality. Postoperative levels of IL6 and TNF $\alpha$ tended to be associated with all-cause mortality, whereas no associations were observed for IL10.

We observed no striking differences between associations with preoperative levels compared with postoperative levels and colorectal cancer outcomes. Levels of inflammation markers did not substantially change over time. Together, results indicate that low-grade systemic inflammation is associated with colorectal cancer recurrence (hsCRP) and all-cause mortality, independently of the presence of the primary tumor. Interestingly, the association between preoperative levels of inflammation markers and colorectal cancer outcomes diminished after correction for postoperative levels, while this did not happen the other way around. These results indicate that postoperative levels of inflammation marker levels were more strongly associated with mortality compared with preoperative levels.

To our knowledge, our study is the largest study that assessed the association between systemic levels of several cytokines mechanistically linked to colorectal cancer tumor immunology (5) and colorectal cancer recurrence. We observed a higher risk of recurrence with higher preoperative as well as postoperative hsCRP levels (median preoperative levels $=2.5 \mu \mathrm{g} / \mathrm{mL} ; \mathrm{IQR}=1.2-6.1$ and postoperative levels $=$ $1.8 \mu \mathrm{g} / \mathrm{mL}$; IQR $0.9-4.1$ ). This is in line with results of previous studies. A previous prospective cohort study in 167 patients with colorectal cancer observed a higher risk of recurrence with higher preoperative hsCRP levels ( $>10 \mu \mathrm{g} / \mathrm{mL}$ vs. $<10 \mu \mathrm{g} / \mathrm{mL}$ ), although this was not statistically significant (39). Although we did not observe an association between IL6, TNF $\alpha$, and IL10 and recurrence, four small previous studies observed a higher risk of recurrence with high compared with low IL6 levels $(11,40)$, TNF $\alpha$ levels (11), or IL10 levels (41). Thus, data are still somewhat inconsistent about the associations between cytokine levels and colorectal cancer recurrence 
Table 1. Baseline characteristics of patients with colorectal cancer stratified by tertiles of the combined inflammatory $z$-score ${ }^{a}$.

\begin{tabular}{|c|c|c|c|c|}
\hline & $\begin{array}{l}\text { Total population } \\
N=748\end{array}$ & $\begin{array}{l}\text { Low level of } \\
\text { inflammation (tertile 1) } \\
N=247\end{array}$ & $\begin{array}{l}\text { Moderate level of } \\
\text { inflammation (tertile 2) } \\
\boldsymbol{N}=\mathbf{2 4 8}\end{array}$ & $\begin{array}{l}\text { High level of } \\
\text { inflammation (tertile } 3 \text { ) } \\
N=247\end{array}$ \\
\hline Age (years) & $67.2(62.1-73.4)$ & $65.6(60.3-70.2)$ & $67.0(61.6-73.0)$ & $68.9(64.2-75.2)$ \\
\hline Gender (female) & $245(33)$ & $79(32)$ & $80(32)$ & $84(34)$ \\
\hline $\mathrm{BMI}\left(\mathrm{kg} / \mathrm{m}^{2}\right)$ & $26.8(24.3-29.7)$ & $25.9(23.9-28.9)$ & $27.2(24.8-30.1)$ & $27.6(24.5-30.9)$ \\
\hline Unknown & 3 & 0 & 1 & 2 \\
\hline \multicolumn{5}{|l|}{ Education $^{b}$} \\
\hline Low & 347 (48) & $107(44)$ & $110(46)$ & $128(55)$ \\
\hline Medium & $179(25)$ & $66(27)$ & $58(24)$ & $54(23)$ \\
\hline High & $193(27)$ & $68(28)$ & $70(29)$ & $52(22)$ \\
\hline Unknown & 29 & 6 & 10 & 13 \\
\hline \multicolumn{5}{|l|}{ Smoking habits } \\
\hline Current & $95(13)$ & $31(13)$ & $29(12)$ & $35(15)$ \\
\hline Former & $429(58)$ & $146(60)$ & $143(59)$ & $137(57)$ \\
\hline Never & $212(29)$ & $68(28)$ & $71(29)$ & $70(29)$ \\
\hline Unknown & 12 & 2 & 5 & 5 \\
\hline \multicolumn{5}{|l|}{ Physical activityc } \\
\hline (hours/week) & $11.3(5.0-20.0)$ & $12.6(6.0-21.4)$ & $10.5(4.5-19.5)$ & $10.3(4.0-19.0)$ \\
\hline Unknown & 13 & 3 & 5 & 5 \\
\hline \multicolumn{5}{|l|}{ Dietary factors $^{\mathrm{d}}$} \\
\hline Fruits & $135(57-225)$ & $151(57-226)$ & $114(46-224)$ & $142(71-223)$ \\
\hline Vegetables & $112(71-171)$ & 114 (75-174) & $106(69-165)$ & $114(73-170)$ \\
\hline Fiber & $22(17-27)$ & $22(18-27)$ & $21(17-27)$ & $22(17-27)$ \\
\hline Red meat & $41(25-61)$ & $41(26-61)$ & $42(25-62)$ & $41(24-61)$ \\
\hline Processed meat & $30(13-47)$ & $30(13-48)$ & $30(15-46)$ & $30(12-46)$ \\
\hline Alcohol & $9(1-21)$ & $12(2-23)$ & $9(1-21)$ & $5(0-19)$ \\
\hline Unknown & 17 & 4 & 7 & 6 \\
\hline \multicolumn{5}{|l|}{ Type of cancer } \\
\hline Colon & $491(66)$ & $156(63)$ & $159(64)$ & $174(70)$ \\
\hline \multirow{2}{*}{\multicolumn{5}{|c|}{$\frac{\text { Rectum }}{\text { Tumor stage }}$}} \\
\hline & & & & \\
\hline I & $216(29)$ & $92(37)$ & $65(26)$ & $57(23)$ \\
\hline ॥ & $206(28)$ & $51(21)$ & $68(27)$ & $86(35)$ \\
\hline III & $326(44)$ & $104(42)$ & $115(46)$ & $104(42)$ \\
\hline \multicolumn{5}{|l|}{$\frac{1 \mathrm{II}}{\text { Type of treatment }}$} \\
\hline Only surgery & $415(56)$ & $140(58)$ & $140(57)$ & $132(54)$ \\
\hline Surgery and chemotherapy & $158(21)$ & $48(20)$ & $53(21)$ & $57(23)$ \\
\hline Surgery and radiotherapy & $87(12)$ & $31(13)$ & $29(12)$ & $25(10)$ \\
\hline Surgery and chemoradiation & $71(9)$ & $20(8)$ & $21(9)$ & $29(12)$ \\
\hline Unknown & 16 & 7 & 5 & 4 \\
\hline Use of NSAIDs (yes) & $135(18)$ & $39(16)$ & $56(23)$ & $40(17)$ \\
\hline \multicolumn{5}{|l|}{ Comorbidities } \\
\hline Yes & $561(75)$ & $168(68)$ & $192(78)$ & $197(80)$ \\
\hline Unknown & 2 & 0 & 1 & 1 \\
\hline Serum $25(\mathrm{OH}) \mathrm{D}_{3}$ levels $(\mathrm{nmol} / \mathrm{L})$ & $53.7(40.3-68.9)$ & $57.1(42.4-74.0)$ & $52.5(39.1-67.1)$ & $51.8(38.2-68.0)$ \\
\hline \multicolumn{5}{|l|}{ Season of blood collection ${ }^{\mathrm{e}}$} \\
\hline Spring & $213(28)$ & $78(32)$ & $62(25)$ & $73(30)$ \\
\hline Summer & $220(29)$ & $72(29)$ & $66(27)$ & $80(32)$ \\
\hline Autumn & $138(18)$ & $42(17)$ & $48(19)$ & $47(19)$ \\
\hline Winter & $177(24)$ & $55(22)$ & $72(29)$ & $47(19)$ \\
\hline \multicolumn{5}{|l|}{ Inflammatory markers } \\
\hline IL6 (pg/mL) & $1.0(0.7-1.6)$ & $0.7(0.5-0.9)$ & $1.0(0.8-1.4)$ & $1.8(1.2-3.1)$ \\
\hline IL8 (pg/mL) & $5.7(4.3-8.1)$ & $4.3(3.3-5.4)$ & $5.6(4.2-7.4)$ & $8.7(6.5-12.9)$ \\
\hline IL10 (pg/mL) & $0.2(0.2-0.3)$ & $0.2(0.1-0.2)$ & $0.2(0.2-0.3)$ & $0.3(0.3-0.6)$ \\
\hline $\mathrm{TNF}(\mathrm{pg} / \mathrm{mL})$ & $2.1(1.6-2.6)$ & $1.6(1.3-1.9)$ & $2.0(1.7-2.5)$ & $2.6(2.2-3.3)$ \\
\hline $\mathrm{CRP}(\mu \mathrm{g} / \mathrm{mL})$ & $2.5(1.2-6.1)$ & $1.1(0.6-2.0)$ & $2.5(1.4-4.1)$ & $7.8(3.6-16.9)$ \\
\hline Combined & -0.08 & -0.60 & -0.08 & 0.62 \\
\hline Inflammatory z-score & $(-0.44-0.38)$ & $(-0.81$ to -0.44$)$ & $(-0.21-0.05)$ & $(0.40-0.98)$ \\
\hline
\end{tabular}

(Continued on the following page) 
Wesselink et al.

Table 1. Baseline characteristics of patients with colorectal cancer stratified by tertiles of the combined inflammatory $z$-score ${ }^{\mathrm{a}}$. (Cont'd)

\begin{tabular}{lllll}
\hline & $\begin{array}{l}\text { Total population } \\
\mathbf{N = 7 4 8}\end{array}$ & $\begin{array}{l}\text { Low level of } \\
\text { inflammation (tertile 1) } \\
\boldsymbol{N}=\mathbf{2 4 7}\end{array}$ & $\begin{array}{l}\text { Moderate level of } \\
\text { inflammation (tertile 2) } \\
\boldsymbol{N}=\mathbf{2 4 8}\end{array}$ & $\begin{array}{l}\text { High level of } \\
\text { inflammation (tertile 3) } \\
\boldsymbol{N}=\mathbf{2 4 7}\end{array}$ \\
\hline $\begin{array}{l}\text { Cohort } \\
\text { COLON }\end{array}$ & & & $144(58)$ \\
EnCoRe & $487(65)$ & $172(70)$ & $169(68)$ & $103(42)$ \\
\hline Deceased & $261(35)$ & $75(30)$ & $79(32)$ & $42(17)$ \\
\hline Recurrence & $98(13)$ & $20(8)$ & $35(14)$ & $30(12)$ \\
\hline
\end{tabular}

Note: Values presented are median (quartile 1-quartile 3) or number (percentages).

aLow, moderate, and high levels of inflammation were derived from the tertiles of the combined inflammatory $z$-scores of IL $6+$ IL $8+$ IL10 + TNF $\alpha+$ CRP $/ 5$. For 81 patients, levels of one inflammation marker were missing. For these patients, the main inflammatory $z$-score was calculated by summing the $z$-scores of the four available inflammation markers/4. For five patients, more than one inflammatory marker was missing. For these patients, no mean inflammatory $z$-score was calculated. Cut-off points for the tertiles of the total inflammation level were -0.32 and 0.18

bLow education was defined as primary school and lower general secondary education; medium as lower vocational training and higher general secondary education; high as high vocational training and university.

${ }^{\mathrm{c} A c t i v i t i e s}$ with a metabolic equivalent score (MET score) $\geq 3$ were defined as moderate to vigorous physical activity.

dintake in grams per day.

eSpring: March-May; Summer: June-August; Autumn: September-November; Winter: December-February.

and more research is needed to further elucidate the role of inflammatory cytokines in colorectal cancer recurrence. To further explore this, future research should investigate local inflammation in the tumor microenvironment, as well as circulating levels, preferably several times, before, during, and after treatment $(42,43)$.

All proinflammatory markers (IL6, IL8, TNF $\alpha$, hsCRP) measured tended to be associated with a higher risk of all-cause mortality, but this was only statistically significant for IL8 and the combined inflammatory $z$-score. No associations were observed for the anti-inflammatory cytokine IL10. The higher risk of all-cause mortality with higher IL8 levels is in line with results of previous studies (20). In a meta-analysis including four studies with in total 330 patients, high preoperative serum IL8 levels compared with low levels were associated with a higher risk of all-cause mortality (pooled HR $=2.13$; 95\% CI, 1.493.05 ; ref. 20). Most previous studies observed a statistically significant association between preoperative IL6 and TNF $\alpha$ in relation to all-cause mortality $(14,17,19)$. In a meta-analysis including six studies with in total 679 patients with CRC high versus low levels of IL6 were associated with a higher risk of all-cause mortality (pooled HR = 1.76; 95\% CI, 1.42-2.19; ref. 17). In a study in 119 patients with colorectal cancer, a significant longer median survival time was observed in patients with high versus low levels of TNF $\alpha$ (19), whereas a small study in 74 patients with colorectal cancer with local disease did not observe an association between TNF and mortality (44). Regarding CRP, a meta-analysis including 21 studies with a total of 3,934 patients with CRC observed a pooled HR of 2.04 (95\% CI, 1.45-2.86) for allcause mortality, comparing high preoperative CRP levels with low levels (7), which is in line with results of our study. Although we did not observe an association between IL10 and all-cause mortality, a previous study observed a worse survival rate with higher postdiagnostic IL10 levels (41). Combining the results of our and previous studies, we can conclude that, in general, higher preoperative and postoperative levels of proinflammatory markers are associated with worse colorectal cancer survival.
A

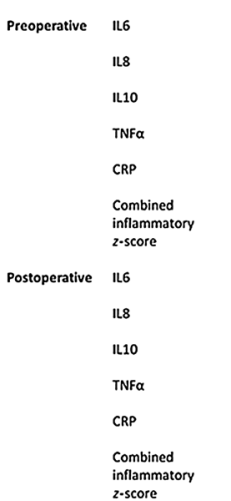

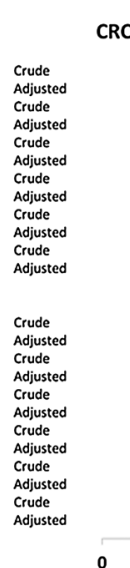

CRC recurrence

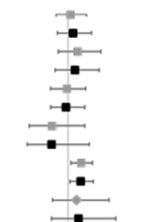

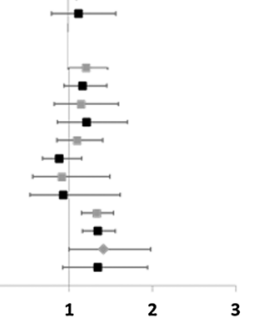

B

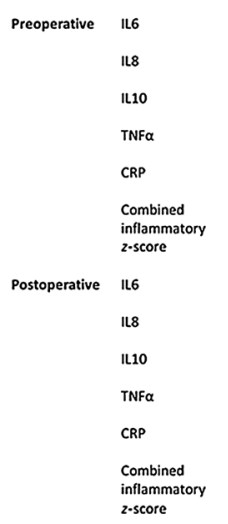

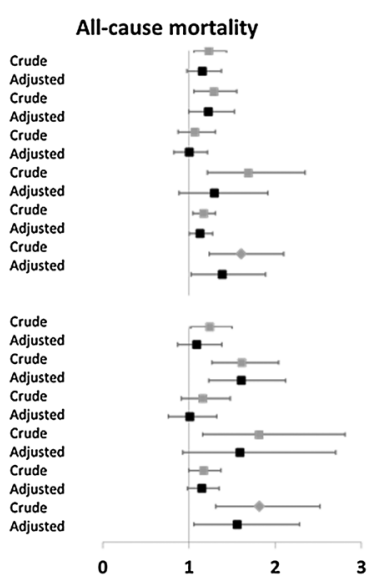

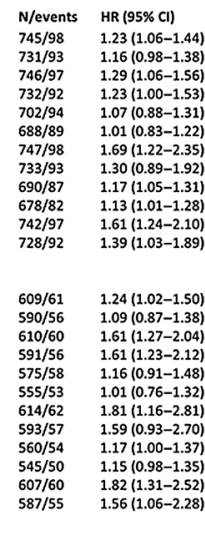

Figure 2.

The association between preoperative and postoperative levels of inflammation markers and colorectal cancer recurrence (A) and all-cause mortality (B). The adjusted models were adjusted for age, sex, BMI, stage of disease, smoking status, cohort, and season of blood collection. Overall inflammation was assessed by calculating a mean inflammatory $z$-score. First, a normalized $z$-score for each individual inflammation marker was calculated as $z=(x-\mu) / \sigma$. The combined score was calculated by summing the $z$-scores of each individual marker and dividing the total sum by the number of inflammation markers included. If data of two or more inflammatory markers were missing ( $n=6$ and 7$)$, no combined inflammatory $z$-score was calculated. 


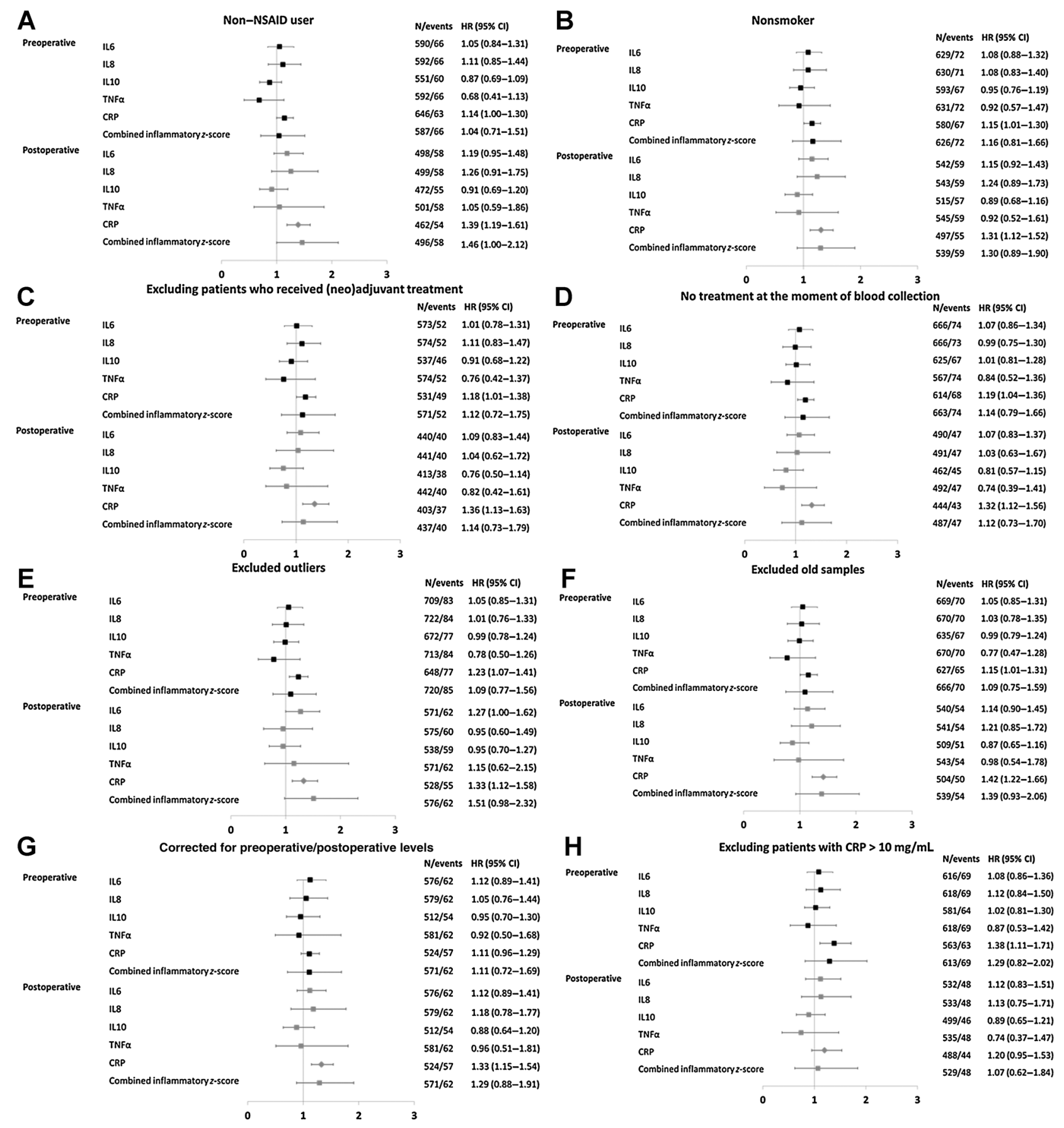

Figure 3.

Results of sensitivity analyses regarding the association between inflammation markers and recurrence, adjusted for age, sex, BMI, stage of disease, smoking status, cohort, and season of blood collection. Sensitivity analyses were done (i) for only NSAID nonusers (A), (ii) for only nonmokers (B), (iii) excluding patients who received (neo)adjuvant treatment (for preoperative levels, patients who received neoadjuvant treatment were excluded; for postoperative levels, patients who received adjuvant treatment were excluded) (C). (iv) excluding patients still undergoing treatment (for preoperative levels, patients who already started treatment were excluded; for postoperative levels, patients who still underwent adjuvant treatment were excluded) (D), (v) excluding outliers (biomarkers more than 3 SD below or above the mean) (E), (vi) excluding old samples (levels measured after two years of storage) (F), (vii) correcting for preoperative or postoperative levels (G), and (viii) excluding patients with hsCRP levels $>10 \mathrm{mg} / \mathrm{mL}$ (H).

The mechanisms by which inflammation as reflected by aberrant expression of cytokines promotes colorectal cancer progression have extensively been studied in in vitro and animal studies $(5,45)$. Several interleukins, including IL6, IL8, and IL10, have tumor-promoting effects. IL6 has several important roles in cancer progression, as it stimulates processes such as cell proliferation, migration, and 
Wesselink et al.
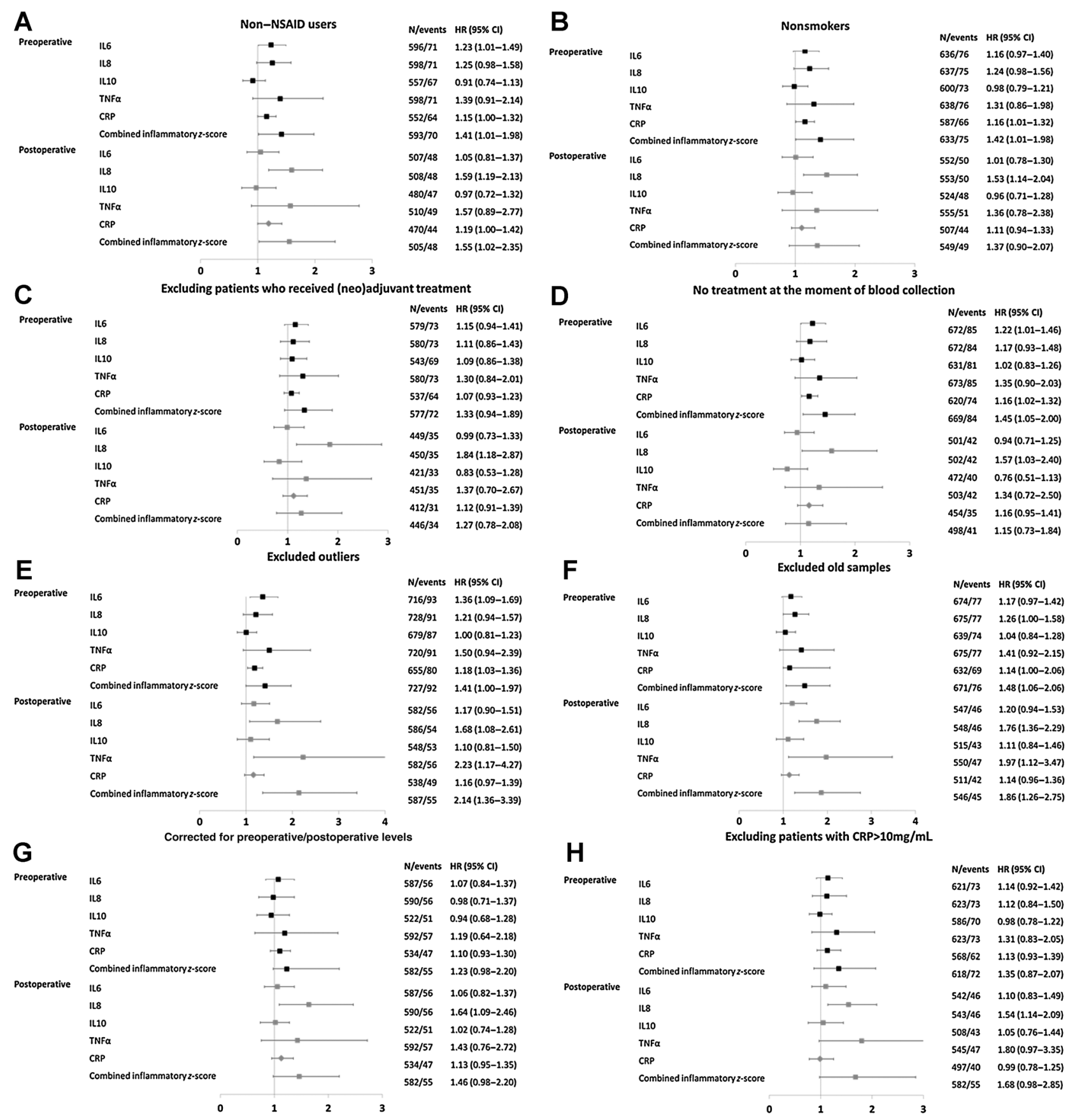

Figure 4.

Results of sensitivity analyses regarding the association between inflammation markers and all-cause mortality, adjusted for age, sex, BMI, stage of disease, smoking status, cohort, and season of blood collection. Sensitivity analyses were done (i) for only NSAID nonusers (A), (ii) for only nonsmokers (B), (iii) excluding patients who received (neo)adjuvant treatment (for preoperative levels, patients who received neoadjuvant treatment were excluded; for postoperative levels, patients who received adjuvant treatment were excluded) (C), (iv) excluding patients still undergoing treatment (for preoperative levels, patients who already started treatment were excluded; for postoperative levels, patients who still underwent adjuvant treatment were excluded) (D), (v) excluding outliers (biomarkers more than 3 SD below or above the mean) (E), (vi) excluding old samples (levels measured after 2 years of storage) (F), (vii) correcting for preoperative or postoperative levels (G), and (viii) excluding patients with hsCRP levels $>10 \mathrm{mg} / \mathrm{mL}(\mathbf{H})$.

angiogenesis. IL6 mainly exerts is tumor-promoting effects by activating STAT3 $(5,45)$. IL8 mainly promotes colorectal cancer progression through the activation of intercellular signaling PI3K and Akt. The PI3k/Akt pathway plays an essential role in cell survival, migra- tion, and angiogenesis (45). How IL10 is related to colorectal cancer progression is less clear. Some research suggest a tumor promoting effect, whereas other studies propose a tumor inhibitory effect $(18,45,46)$. In addition to ILs, TNFo is also an important 
cytokine that mainly drives tumor growth by activating NF- $\kappa \mathrm{B}$ (5). Finally, CRP plays important roles in inflammatory processes, apoptosis, and the production of cytokines, particularly IL6 and TNF $\alpha$ (47), which in turn, stimulates colorectal cancer progression. Thus, all inflammatory markers investigated in this study are mechanistically linked to colorectal cancer progression. Although IL6 and TNF $\alpha$ also seems to be associated with all-cause mortality, we observed the strongest associations for IL8 and hsCRP. One reason for this could be the variability in levels. The variation in plasma levels of IL6, IL10, and TNF $\alpha$ was low [median $(\mathrm{IQR})=1.0(0.7-1.6), 0.2(0.2-0.3)$, and 2.1 (1.6-2.6), respectively], whereas more variation was observed in hsCRP and IL8 levels [median (IQR) = 5.7 (4.3-8.1) and 2.5 (1.2-6.1), respectively].

We also investigated whether lifestyle factors influenced the associations between levels of inflammatory markers and cancer outcomes. In our study only smoking status and BMI influenced the association between levels of inflammatory markers and cancer outcomes. Thus, although many of these lifestyle factors potentially influence both inflammation and survival (21-26), these lifestyle factors did not substantially influence the association between inflammation and recurrence and all-cause mortality in patients with colorectal cancer in our study. One possible explanation for this could be that our participants were relatively health conscious, resulting in relatively low variation in dietary intake and physical activity levels.

An integrative evaluation of (i) systemic inflammation, (ii) detailed profiling of inflammation and host immunity in the tumor microenvironment, and (iii) factors influencing inflammation is important to extend our knowledge on inflammation in relation to cancer recurrence and survival. This molecular pathological epidemiological approach $(42,43)$ is essential to further unravel such complex interrelationships. Thus, as a next step, local inflammation at the tumorsite, the so-called tumor microenvironment, should be examined to further elucidate the role of inflammation in colorectal cancer recurrence and mortality. Special attention should be given to cytokines known to be involved in tumor progression such as IL6, IL8, and TNF $\alpha(5,45)$, and to immune cell infiltration (48) rather than on more general markers of inflammation, such as CRP. Finally, clinical and lifestyle factors should also be examined as they could potentially influence inflammation and could therefore modify the association between inflammation and colorectal cancer outcomes.

Our study has several strengths. First, we examined a set of cytokines suggested to play an important role in colorectal cancer prognosis before and after surgery. Furthermore, our study is one of the first studies that investigated the association between cytokines and colorectal cancer recurrence. In addition, previous studies assessed the association between inflammation markers and cancer outcomes using inflammation markers as a categorical exposure variable. However, it is debated whether categorization of a continuous variable is always appropriate (49). In our study, we investigated inflammation markers as continuous variables. Besides, we also performed restricted cubic spline analyses to get more insight into the relationship between inflammation marker levels and cancer outcomes. Thus, results of our study extend existing evidence regarding the association between inflammation and colorectal cancer outcomes.

Our study also has some limitations. First, the absolute levels and the variation in levels of the inflammation markers were low. For example, the median IL6 levels was $1.0 \mathrm{pg} / \mathrm{mL}(\mathrm{IQR}=0.7-1.6)$. The low variance in the data limit the ability to detect associations. Still, we observed statistically significant associations between several inflammation markers and cancer outcomes. Second, it would be of interest to investigate whether the association between inflammation markers and mortality is cancer specific or more related to other comorbid conditions such as cardiometabolic diseases. This could be of importance because chronic inflammation is also suggested to play a role in the onset of comorbidities such as cardiovascular diseases and diabetes $(27,28)$. Unfortunately, we had no data available about the cause of death, due to strict privacy regulations in the Netherlands. Therefore, we were not able to perform analyses with disease-specific mortality as an outcome.

In conclusion, preoperative and postoperative levels of several inflammation markers were associated with an increased risk of recurrence and/or all-cause mortality in stage I to III patients with colorectal cancer. The complex role of inflammation in cancer recurrence should be further elucidated using a molecular pathological epidemiology approach.

\section{Authors' Disclosures}

M. van Zutphen reports grants from Dutch Cancer Society outside the submitted work. J.H.W. de Wilt reports grants from Dutch Cancer Society, ZONMW, Medtronic, and Bergh in het Zadel Foundation outside the submitted work. No disclosures were reported by the other authors.

\section{Data Availability}

Since the data consist of identifying cohort information, some access restrictions apply and therefore cannot be made publicly available. Data will be shared with permission from the acting committee of the COLON Study. Requests for data can be sent to Dr. Fränzel van Duijnhoven, Division of Human Nutrition and Health, Wageningen University \& Research, the Netherlands (e-mail: franzel.vanduijnhoven@wur.nl). Requests for data of the EnCoRe study can be sent to Dr. Martijn Bours, Department of Epidemiology, GROW-School for Oncology and Developmental Biology, Maastricht University, the Netherlands (e-mail: m.bours@maastrichtuniversity.nl).

\section{Authors' Contributions}

E. Wesselink: Conceptualization, resources, data curation, formal analysis, visualization, methodology, writing-original draft, project administration, writing-review and editing. M.G.J. Balvers: Conceptualization, resources, data curation, writingreview and editing. D.E. Kok: Resources, writing-review and editing. R.M. Winkels Resources, writing-review and editing. M. van Zutphen: Data curation, writingreview and editing. R.W.M. Schrauwen: Resources, writing-review and editing. E.T.P. Keulen: Resources, writing-review and editing. E.A. Kouwenhoven Resources, writing-review and editing. S.O. Breukink: Resources, writing-review and editing. R.F. Witkamp: Conceptualization, writing-review and editing J.H.W. de Wilt: Conceptualization, resources, writing-review and editing M.J.L. Bours: Conceptualization, supervision, writing-review and editing. M.P. Weijenberg: Conceptualization, funding acquisition, writing-review and editing. E. Kampman: Conceptualization, funding acquisition, writing-review and editing. F.J.B. van Duijnhoven: Conceptualization, supervision, funding acquisition, writing-review and editing.

\section{Acknowledgments}

The authors would like to thank the participants of the COLON study and the investigators at Wageningen University \& Research and the coworkers from the following hospitals for their involvement in recruitment for the COLON study: Hospital Gelderse Vallei, Ede; Radboudumc, Nijmegen; Slingeland Hospital, Doetinchem, Canisius Wilhelmina Hospital, Nijmegen; Rijnstate Hospital, Arnhem; Gelre Hospitals, Apeldoorn/Zutphen; Hospital Bernhoven, Uden; Isala, Zwolle; ZGT, Almelo; Martini Hospital, Groningen; Admiraal de Ruyter Hospital, Goes/Vlissingen. We would like to thank all participants of the EnCoRe study and the health professionals in the three hospitals involved in the recruitment of participants of the study: Maastricht University Medical Center, VieCuri Medical Center, and Zuyderland Medical Center. We would also like to thank the MEMIC center for data and information management and for facilitating the logistic processes and data management of our study. Furthermore, we would like to thank the research dieticians and research assistant who are responsible for patient inclusion and follow-up, performing home visits, as well as data collection and processing. Finally, the authors would like to thank BEVITAL, Bergen, Norway, for analyzing hsCRP in the COLON and EnCoRe study. The COLON study was financially supported by Wereld Kanker 
Onderzoek Fonds, including funds from grant 2014/1179 as part of the World Cancer Research Fund International Regular Grant Programme; Alpe d'Huzes/Dutch Cancer Society (UM 2012-5653, UW 2013-5927, UW 2015-7946); and ERA-NET on Translational Cancer Research (TRANSCAN/Dutch Cancer Society: UW20136397, UW2014-6877). The EnCoRe study was supported by a grant from the Stichting Alpe d'HuZes within the research program 'Leven met kanker' of the Dutch Cancer Society (Grant No. UM-2010-4867) and by a grant from Kankeronderzoekfonds Limburg as part of Health Foundation Limburg (Grant No. 00005739).

\section{References}

1. Hanahan D, Weinberg RA. Hallmarks of cancer: the next generation. Cell 2011; 144:646-74.

2. Lasry A, Zinger A, Ben-Neriah Y. Inflammatory networks underlying colorectal cancer. Nat Immunol 2016;17:230.

3. Grivennikov SI, Greten FR, Karin M. Immunity, inflammation, and cancer. Cell 2010;140:883-99.

4. Shalapour S, Karin M. Immunity, inflammation, and cancer: an eternal fight between good and evil. J Clin Invest 2015;125:3347-55.

5. West NR, McCuaig S, Franchini F, Powrie F. Emerging cytokine networks in colorectal cancer. Nat Rev Immunol 2015;15:615-29.

6. Coyle C, Cafferty FH, Langley RE. Aspirin and colorectal cancer prevention and treatment: is it for everyone? Curr Colorectal Cancer Rep 2016;12:27-34.

7. Woo HD, Kim K, Kim J. Association between preoperative C-reactive protein level and colorectal cancer survival: a meta-analysis. Cancer Causes Control 2015;26:1661-70.

8. Shrotriya S, Walsh D, Bennani-Baiti N, Thomas S, Lorton C. C-Reactive protein is an important biomarker for prognosis tumor recurrence and treatment response in adult solid tumors: a systematic review. PLoS One 2015;10:e0143080.

9. Matsubara D, Arita T, Nakanishi M, Kuriu Y, Murayama Y, Kudou M, et al. The impact of postoperative inflammation on recurrence in patients with colorectal cancer. Int J Clin Oncol 2020;25:602-13.

10. Guthrie GJK, Roxburgh CSD, Horgan PG, McMillan DC. Does interleukin-6 link explain the link between tumour necrosis, local and systemic inflammatory responses and outcome in patients with colorectal cancer? Cancer Treat Rev 2013;39:89-96.

11. Chang PH, Pan YP, Fan CW, Tseng WK, Huang JS, Wu TH, et al. Pretreatment serum interleukin- $1 \beta$, interleukin- 6 , and tumor necrosis factor- $\alpha$ levels predict the progression of colorectal cancer. Cancer Med 2016;5:426-33.

12. Rasic I, Radovic S, Aksamija G. Relationship between chronic inflammation and the stage and histopathological size of colorectal carcinoma. Med Arch 2016; 70:104.

13. Szkaradkiewicz A, Marciniak R, Chudzicka-Strugaøa I, Wasilewska A, Drews M, Majewski P, et al. Proinflammatory cytokines and IL-10 in inflammatory bowel disease and colorectal cancer patients. Arch Immunol Ther Exp 2009;57:291.

14. Knupfer H, Preiss R. Serum interleukin-6 levels in colorectal cancer patients-a summary of published results. Int J Colorectal Dis 2010;25:135-40.

15. Bălăşoiu M, Bălăş oiu AT, Mogoantă SS, Bărbălan A, Stepan AE, Ciurea RN, et al. Serum and tumor microenvironment IL- 8 values in different stages of colorectal cancer. Rom J Morphol Embryol 2014;55:575-8.

16. Seruga B, Zhang H, Bernstein LJ, Tannock IF. Cytokines and their relationship to the symptoms and outcome of cancer. Nat Rev Cancer 2008;8:887.

17. Xu J, Ye Y, Zhang H, Szmitkowski M, Mäkinen M, Li P, et al. Diagnostic and prognostic value of serum interleukin-6 in colorectal cancer. Medicine 2016; 95:e2502.

18. Abtahi S, Davani F, Mojtahedi Z, Hosseini SV, Bananzadeh A, Ghaderi A. Dual association of serum interleukin-10 levels with colorectal cancer. J Cancer Res Ther 2017;13:252.

19. Stanilov N, Miteva L, Dobreva Z, Stanilova S. Colorectal cancer severity and survival in correlation with tumour necrosis factor-alpha. Biotechnol Biotechnol Equip 2014;28:911-7.

20. Xia W, Chen W, Zhang Z, Wu D, Wu P, Chen Z, et al. Prognostic value, clinicopathologic features and diagnostic accuracy of interleukin-8 in colorectal cancer: a meta-analysis. PLoS One 2015;10:e0123484.

21. Wedell-Neergaard A-S, Eriksen L, Grønbæk M, Pedersen BK, Krogh-Madsen R, Tolstrup J. Low fitness is associated with abdominal adiposity and low-grade inflammation independent of BMI. PLoS One 2018;13:e0190645.

22. Calder PC, Ahluwalia N, Brouns F, Buetler T, Clement K, Cunningham K, et al. Dietary factors and low-grade inflammation in relation to overweight and obesity. Br J Nutr 2011;106:S1-S78.
The costs of publication of this article were defrayed in part by the payment of page charges. This article must therefore be hereby marked advertisement in accordance with 18 U.S.C. Section 1734 solely to indicate this fact.

Received December 11, 2020; revised February 19, 2021; accepted March 16, 2021; published first March 26, 2021.

23. Yanbaeva DG, Dentener MA, Creutzberg EC, Wesseling G, Wouters EF. Systemic effects of smoking. Chest 2007;131:1557-66.

24. Meyerhardt JA, Giovannucci EL, Holmes MD, Chan AT, Chan JA, Colditz GA, et al. Physical activity and survival after colorectal cancer diagnosis. J Clin Oncol 2006;24:3527-34.

25. Meyerhardt JA, Niedzwiecki D, Hollis D, Saltz LB, Hu FB, Mayer RJ, et al Association of dietary patterns with cancer recurrence and survival in patients with stage III colon cancer. JAMA 2007;298:754-64.

26. Pelser C, Arem H, Pfeiffer RM, Elena JW, Alfano CM, Hollenbeck AR, et al. Prediagnostic lifestyle factors and survival after colon and rectal cancer diagnosis in the National Institutes of Health (NIH)-AARP Diet and Health Study. Cancer 2014;120:1540-7.

27. Alfano CM, Peng J, Andridge RR, Lindgren ME, Povoski SP, Lipari AM, et al. Inflammatory cytokines and comorbidity development in breast cancer survivors versus noncancer controls: evidence for accelerated aging? J Clin Oncol 2017;35:149.

28. Lopez-Candales A, Burgos PMH, Hernandez-Suarez DF, Harris D. Linking chronic inflammation with cardiovascular disease: from normal aging to the metabolic syndrome. J Nat Sci 2017;3:e341.

29. van Harten-Gerritsen AS, Balvers MG, Witkamp RF, Kampman E, van Duijnhoven FJ. Vitamin D, inflammation and colorectal cancer progression: a review of mechanistic studies and future directions for epidemiological studies. Cancer Epidemiol Biomarkers Prev 2015;24:1820-8.

30. Wesselink E, Balvers M, Bours MJL, de Wilt JHW, Witkamp RF, van Baar H, et al. The association between circulating levels of vitamin $\mathrm{D}$ and inflammatory markers in the first 2 years after colorectal cancer diagnosis. Therap Adv Gastroenterol 2020;13:1756284820923922.

31. Winkels RM, Heine-Broring RC, van Zutphen M, van Harten-Gerritsen S, Kok DE, van Duijnhoven FJ, et al. The COLON study: Colorectal cancer: Longitudinal, Observational study on nutritional and lifestyle factors that may influence colorectal tumour recurrence, survival and quality of life. BMC Cancer 2014;14:374.

32. van Roekel EH, Bours MJ, de Brouwer CP, Ten Napel H, Sanduleanu S, Beets GL et al. The applicability of the international classification of functioning, disability, and health to study lifestyle and quality of life of colorectal cancer survivors. Cancer Epidemiol Biomarkers Prev 2014;23:1394-405.

33. Meyer K, Ueland PM. Targeted quantification of C-reactive protein and cystatin $c$ and its variants by immuno-MALDI-MS. Anal Chem 2014;86:5807-14.

34. Hopkins MH, Owen J, Ahearn T, Fedirko V, Flanders WD, Jones DP, et al. Effects of supplemental vitamin D and calcium on biomarkers of inflammation in colorectal adenoma patients: a randomized, controlled clinical trial Cancer Prev Res 2011;4:1645-54

35. Wendel-Vos GC, Schuit AJ, Saris WH, Kromhout D. Reproducibility and relative validity of the short questionnaire to assess health-enhancing physical activity. J Clin Epidemiol 2003;56:1163-9.

36. Van Leersum N, Snijders H, Henneman D, Kolfschoten N, Gooiker G, Ten Berge M. The Dutch surgical colorectal audit. Eu J Surg Oncol 2013; 39:1063-70.

37. Khoo AL, Chai L, Koenen H, Sweep F, Joosten I, Netea M, et al. Regulation of cytokine responses by seasonality of vitamin D status in healthy individuals. Clin Exp Immunol 2011;164:72-9.

38. de Jager W, Bourcier K, Rijkers GT, Prakken BJ, Seyfert-Margolis V. Prerequisites for cytokine measurements in clinical trials with multiplex immunoassays. BMC Immunol 2009;10:52.

39. Takasu C, Shimada M, Kurita N, Iwata T, Nishioka M, Morimoto S, et al. Impact of C-reactive protein on prognosis of patients with colorectal carcinoma. Hepatogastroenterology 2013;60:507-11.

40. Hara M, Nagasaki T, Shiga K, Takahashi H, Takeyama H. High serum levels of interleukin- 6 in patients with advanced or metastatic colorectal cancer: the effect 
Inflammation Marker Levels and Colorectal Cancer Outcomes

on the outcome and the response to chemotherapy plus bevacizumab. Surg Today 2017;47:483-9.

41. Li B, Wang F, Ma C, Hao T, Geng L, Jiang H. Predictive value of IL-18 and IL-10 in the prognosis of patients with colorectal cancer. Oncol Lett 2019;18:713-9.

42. Ogino S, Campbell PT, Nishihara R, Phipps AI, Beck AH, Sherman ME, et al Proceedings of the second international molecular pathological epidemiology (MPE) meeting. Cancer Causes Control 2015;26:959-72.

43. Ogino S, Chan AT, Fuchs CS, Giovannucci E. Molecular pathological epidemiology of colorectal neoplasia: an emerging transdisciplinary and interdisciplinary field. Gut 2011;60:397-411.

44. Nikiteas NI, Tzanakis N, Gazouli M, Rallis G, Daniilidis K, Theodoropoulos G et al. Serum IL-6, TNFalpha and CRP levels in Greek colorectal cancer patients: prognostic implications. World J Gastroenterol 2005;11:1639-43.
45. Li J, Huang L, Zhao H, Yan Y, Lu J. The role of interleukins in colorectal cancer Int J Biol Sci 2020;16:2323-39.

46. Mannino MH, Zhu Z, Xiao H, Bai Q, Wakefield MR, Fang Y. The paradoxical role of IL-10 in immunity and cancer. Cancer Lett 2015;367:103-7.

47. Sproston NR, Ashworth JJ. Role of C-reactive protein at sites of inflammation and infection. Front Immunol 2018;9:754.

48. Pagès F, Mlecnik B, Marliot F, Bindea G, Ou F-S, Bifulco C, et al. International validation of the consensus Immunoscore for the classification of colon cancer: a prognostic and accuracy study. Lancet North Am Ed 2018; 391:2128-39.

49. Bennette C, Vickers A. Against quantiles: categorization of continuous variables in epidemiologic research, and its discontents. BMC Med Res Method 2012;12:21. 


\section{Cancer Epidemiology, Biomarkers \& Prevention}

\section{Levels of Inflammation Markers Are Associated with the Risk of Recurrence and All-Cause Mortality in Patients with Colorectal Cancer}

Evertine Wesselink, Michiel G.J. Balvers, Dieuwertje E. Kok, et al.

Cancer Epidemiol Biomarkers Prev 2021;30:1089-1099. Published OnlineFirst March 26, 2021.

\section{Updated version Access the most recent version of this article at:} doi:10.1158/1055-9965.EPI-20-1752

Supplementary Access the most recent supplemental material at:

Material http://cebp.aacrjournals.org/content/suppl/2021/03/20/1055-9965.EPI-20-1752.DC1

Cited articles This article cites 49 articles, 5 of which you can access for free at: http://cebp.aacrjournals.org/content/30/6/1089.full\#ref-list-1

E-mail alerts Sign up to receive free email-alerts related to this article or journal.

Reprints and To order reprints of this article or to subscribe to the journal, contact the AACR Publications Department Subscriptions at pubs@aacr.org.

Permissions To request permission to re-use all or part of this article, use this link http://cebp.aacrjournals.org/content/30/6/1089.

Click on "Request Permissions" which will take you to the Copyright Clearance Center's (CCC) Rightslink site. 